\title{
A Conidial Actinoplanes Isolate from Blelham Tarn
}

\author{
By L. G. WILLOUGHBY \\ Freshwater Biological Association, Ambleside, Westmorland
}

(Received 9 November 1965)

\section{SUMMARY}

An Actinoplanes sp. (Actinomycetales) isolated from Blelham Tarn is described and illustrated. It is characterized by a filed arrangement of spores both in sporangia and conidiophore systems, suggesting that these two types of reproductive structure may develop in an essentially similar manner.

\section{INTRODUCTION}

Actinomycetales bearing sporangia were first reported by Couch in 1949 and placed in a special family, the Actinoplanaceae. Subsequently Couch $(1955,1963)$ distinguished several constituent genera of which Actinoplanes has been represented the most frequently in his isolations, which have largely been from soil. The purpose of this contribution is to describe an actinoplanes isolate which has several interesting morphological features; in particular it bears striking conidiophore systems in addition to sporangia.

\section{METHODS AND RESULTS}

The isolate was obtained by plating and incubating a freshly collected surface water sample from Blelham Tarn (English Lake District) on starch casein agar (Küster \& Williams, 1964). Subcultures on the latter medium were creamy yellow in colour, and with a rather diffuse colony margin. Subcultures were also set up on pinus pollen suspended in sterile lake water. Under these conditions growth of the organism was particularly prolific and clearly displayed and gave most of the observations reported. Sporangial initials occurred at hyphal tips (Fig. 1; Pl. 1, fig. 1) and it was sometimes possible to see some indication of a longitudinal arrangement of contents very early in development (Fig. 3). At maturity the sporangium was normally oblong (Fig. 6) or suburceolate (Pl. 1, figs. 2, 3); a double structure was not unusual (Figs. 7, 9; Pl. 1, fig. 7). As with other actinoplanes isolates which have been studied in this laboratory, dehiscence could usually be induced by mounting ripe material under a coverglass. However, the very rapid sequence of visible sporangiospore differentiation and dehiscence often observed in these cases, particularly well exemplified in an isolate from soil from Kynance Cove, Cornwall (Pl. 2), was not a feature of the Blelham Tarn isolate. In the latter sporangiospore outlines, arranged in a characteristically filed fashion, were distinguished when the material was first mounted (e.g. as in Fig. 4; Pl. 1, fig. 3). There was a more leisurely accentuation and rounding off of these outlines (Fig. 6; Pl. 1, fig. 4) followed by a correspondingly protracted deliquescence of sporangial walls, either partial (Figs. 10, 11) or more or less complete (Fig. 12). Sporangiospores were 
motile, but there was a considerable time lag (up to several hours) before they became highly active. The usual pattern was to observe a background of highly active sporangiospores traversing the microscope field with a foreground of sporangia

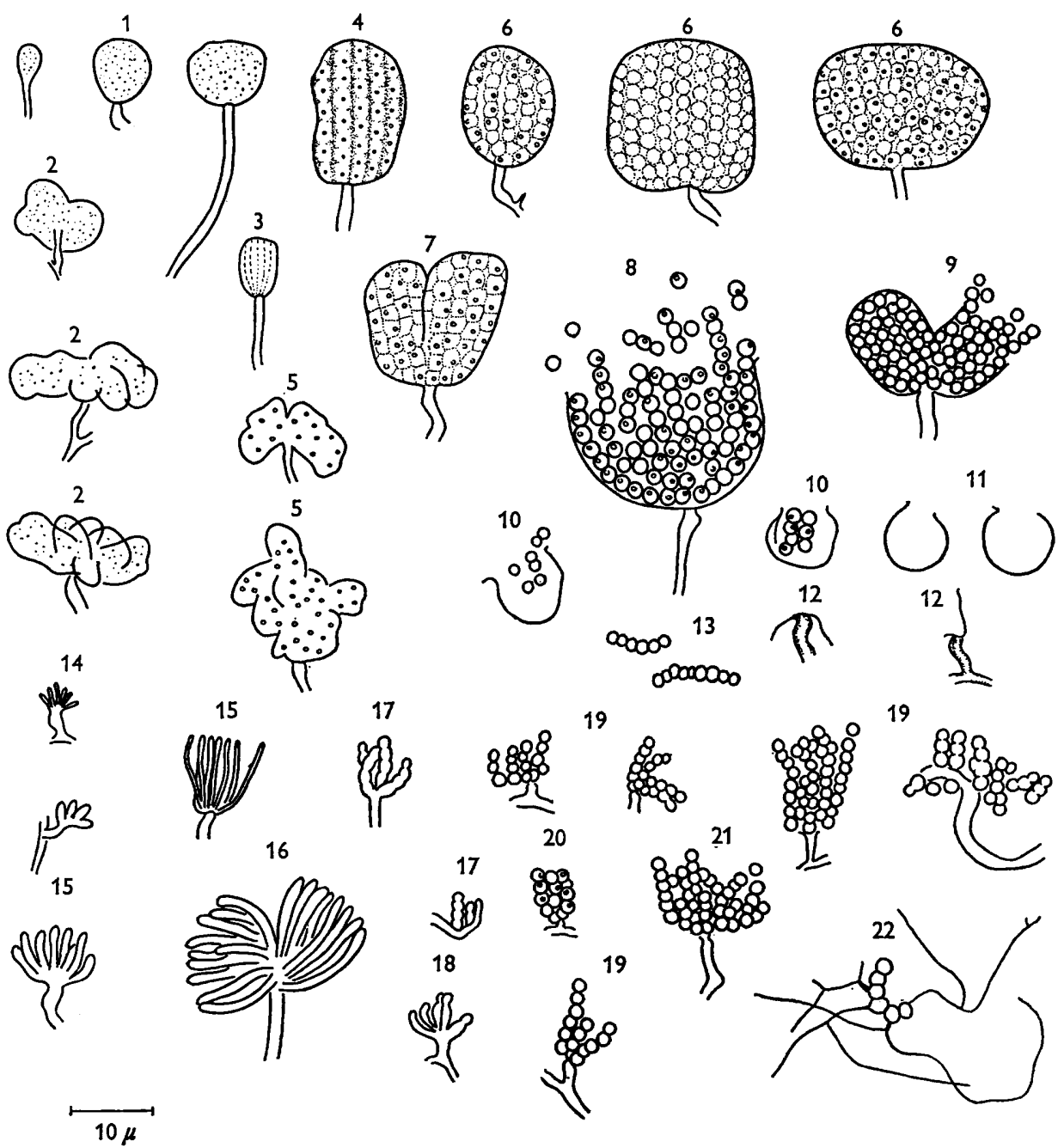

Camera-lucida drawings of Actinoplanes sp. from Blelham Tarn.

Fig. 1, young sporangia. Fig. 2, irregularly shaped specimens occasionally observed. Fig. 3, young sporangium with indication of longitudinal internal arrangement. Fig. 4, sporangium with refractive globules and outlines of sporangiospores first apparent. Fig. 5, a corresponding stage in irregularly shaped sporangia. Fig. 6, sporangiospore outlines more clearly defined. Fig. 7, a double sporangium structure. Figs. 8-10, dehiscence and sporangiospore liberation. Fig. 11, dehisced sporangia. Fig. 12, dehisced sporangia with very little residual wall material apparent. Fig. 13, liberated sporangiospore chains. Fig. 14, young conidiophore systems. Fig. 15, conidiophores following further growth. Fig. 16, conidiophore system at mature size. Fig. 17, conidiospore constriction beginning. Fig. 18, conidiophores with apical constriction only. Fig. 19, mature conidiophore systems. Fig. 20, conidiospores showing refractive globule. Fig. 21, orderly arrangement of conidiospores breaking down. Fig. 22, germination of a chain of sporangiospore on starch casein agar. 
which were dehisced, but the spores in which remained quiescent. These sporangia were difficult to distinguish from conidiophore systems, as described below. The consistent observation of the liberation of pairs or chains of sporangiospores rather than single units (Figs. 8, 10, 13; Pl. 1, figs. 15, 16) gave further confirmation, if any were needed, of the filed arrangement of sporangiospores in the sporangia. These spore chains only exhibited extremely limited motility; the question arose whether they had been squashed out of sporangia prematurely rather than liberated in a normal fashion. The fact that spore chains are viable (Fig. 22; Pl. 1, fig. 17) is deemed to imply that they are in fact a normal phenomenon in this isolate.

Conidiophore systems were distinguished in their earliest stages as clusters of finger-like hyphae radiating from a common stalk (Fig. 14; Pl. 1, figs. 9, 10). The hyphae expanded both in length and girth and eventually superficial constrictions were apparent (Fig. 17). Although the constriction and subsequent separation of conidiospores in a fertile hypha normally proceeded simultaneously along its length, there was evidence that successive separations from the apex were also possible (Fig. 18). At maturity the conidiophore systems consisted of loosely-borne divergent files of conidiospores (Fig. 19; Pl. 1, fig. 14) the orderly arrangement of which tended to break down in the larger examples (Fig. 21).

When subcultures grown on pollen were mounted for microscopic examination mature sporangia and mature conidiophore systems could usually be distinguished initially. However, as the sporangial walls became indistinct at the onset of dehiscence, and this was often accompanied by a loosening of the compact arrangement of the sporangiospores (Pl. 1, fig. 5), it became increasingly difficult to make the distinction. Eventually sporangiospores were distinguishable from conidiospores by their motility, on occasions when this was pronounced. The limits of the size-range of sporangiospores $(1 \cdot 3-1 \cdot 8 \mu)$ were overlapped by those of conidiospores $(1 \cdot 1-1 \cdot 8 \mu)$. The refractive globule which was usually a conspicuous feature of sporangiospores was rarely to be distinguished in conidiospores (Fig. 20). Despite these various points of difference between the two types of sporulation unit and spore the difficulty experienced in making a visual segregation gave acute awareness of their essential similarity.

\section{DISCUSSION}

Although there is a previous report and description of the occurrence of conidia in the genus Actinoplanes (Kalakutskii \& Kuznetsov, 1964) and remarks by other authors imply that this is not unusual, the Blelham Tarn isolate would seem to be the only documented instance so far where a definite conidial system, at maturity outwardly similar to the sporangium, has been reported. In this isolate the liberation of spore chains from the sporangia tends to suggest that the filed arrangement of sporangiospores occurs through the septation of parallel lengths of hyphae. Lechevalier \& Holbert (1965) produced evidence from electron microscope studies that sporangiospores in an actinoplanes isolate made by them were so derived. In comparing the sporangial and conidial systems in the Blelham Tarn isolate it may be deduced that the main essential difference lies in the enclosure of the whole sporing system in a common membrane in the sporangial system only. Clearly such an ontogeny for the sporangium is quite different from that in the lower aquatic fungi (Chambers \& Willoughby, 1964), with which the Actinoplanaceae show such outwardly close affinity. 
My thanks are due to Mrs P. J. McDougall for valuable assistance and to Mr T. Cross of the Bradford Institute of Technology for help with the literature.

\section{REFERENCES}

Couch, J. N. (1955). A new genus and family of the Actinomycetales, with a revision of the genus Actinoplanes. J. Elisha Mitchell scient. Soc. 71, 148.

Couch, J. N. (1963). Some new genera and species of the Actinoplanaceae. J. Elisha Mitchell scient. Soc. 79, 53.

Kalakutskit, L. V. \& Kuznetsov, V. D. (1964). A new species of the genus Actinoplanes Couch, Actinoplanes armeniacus n.sp., and some peculiarities of its mode of spore formation. Mikrobiologiya, 33, 613.

Küster, E. \& Williams, S. T. (1964). Selection of media for isolation of Streptomycetes. Nature, Lond. 202, 928.

Lechevalier, H. \& Holbert, P. E. (1965). Electron microscopic observation of the sporangial structure of a strain of Actinoplanes. J. Bact. 89, 217.

Chambers, T. C. \& Willoughby, L. G. (1964). The fine structure of Rhizophlyctis rosea, a soil Phycomycete. $J l R$. microsc. Soc. 83, 355.

\section{EXPLANATION OF PLATES}

Plate 1

Photomicrographs of Actinoplanes sp. from Blelham Tarn. All $\times 1000$ except Figs. 17 and 18 .

Fig. 1. Young stalked sporangium.

Fig. 2. Field of young sporangia.

Fig. 3. Sporangia with sporangiospores first distinguished.

Fig. 4. Sporangiospores now fully apparent.

Figs. 5, 6. Sporangia with walls deliquesced but zoospores quiescent.

Fig. 7. A double sporangium with wall deliquesced at top right only.

Fig. 8. An irregularly shaped sporangium.

Figs. 9, 10. Conidiophore initials.

Fig. 11. Conidiophore system with oblong conidiospores distinguished.

Fig. 12. Young conidiophore system and sporangium showing close similarity in gross external morphology.

Fig. 13. Conidiophores at full size but conidiospores not constricted.

Fig. 14. Mature conidiospores.

Figs. 15, 16. Chains of sporangiospores.

Fig. 17. Single sporangiospores and a chain (right) germinating on starch casein agar $(\times 2000)$.

Fig. 18. Vertical section of growth on starch casein agar showing strongly marked palisade $(\times 630)$.

\section{Plate 2}

Photomicrographs of Actinoplanes sp. from soil from Kynance Cove, Cornwall showing sequences of sporangiospore differentiation and dehiscence in several sporangia on Pinus pollen. The series runs from left to right, top to bottom, and was made over a period of approximately $20 \mathrm{~min}$. All $\times 630$. 
Journal of General Microbiology, Vol. 44, No. 1

Plate 1

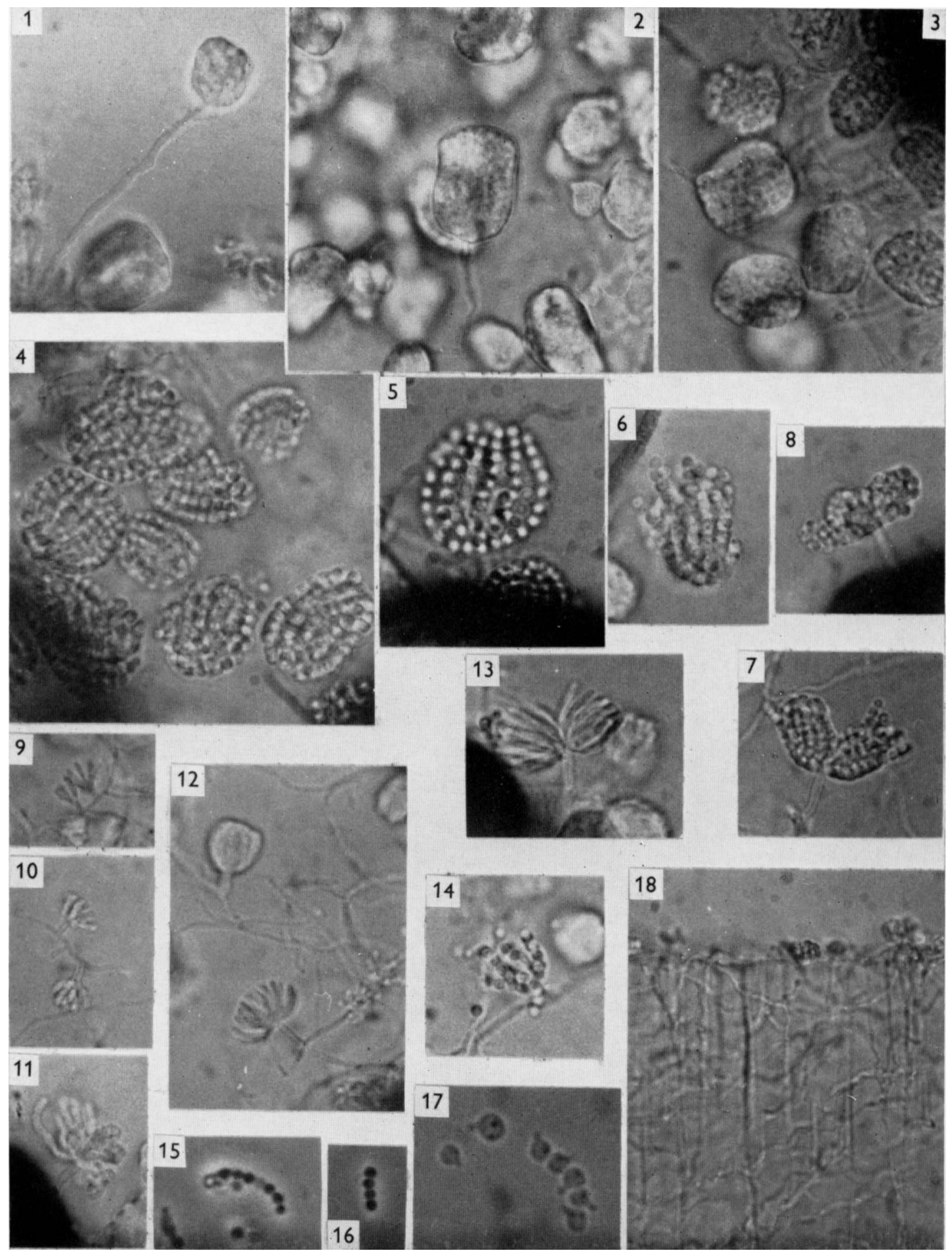

L. G. WILLOUGHBY

(Facing $p .72)$ 
Journal of General Microbiology, Vol. 44, No. 1

Plate 2
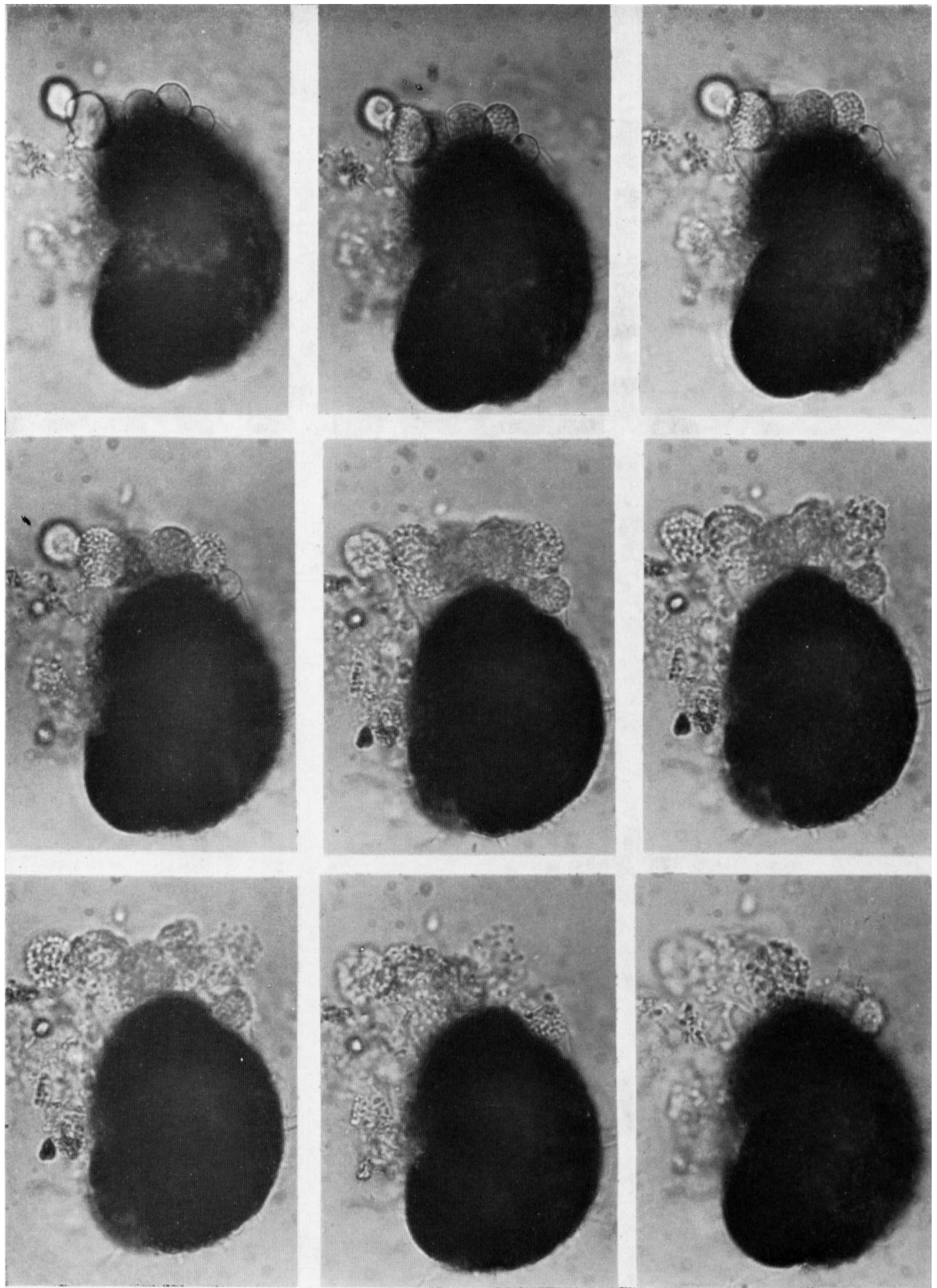

L. G. WILLOUGHBY 